\title{
Magnetic proximity effect in perovskite superconductor/ferromagnet multilayers
}

\author{
J. Stahn, ${ }^{1}$ J. Chakhalian, ${ }^{2}$ Ch. Niedermayer, ${ }^{1}$ J. Hoppler, ${ }^{1}$ T. Gutberlet, ${ }^{1}$ J. Voigt, ${ }^{1}$ F. Treubel, ${ }^{3}$ H-U. Habermeier, ${ }^{2}$ \\ G. Cristiani, ${ }^{2}$ B. Keimer, ${ }^{2}$ and C. Bernhard ${ }^{2}$ \\ ${ }^{1}$ Laboratorium für Neutronenstreuung, ETH Zürich \& PSI, Villigen, Switzerland \\ ${ }^{2}$ Max Planck Institut für Festkörperforschung, Stuttgart, Germany \\ ${ }^{3}$ Fakultät für Physik, Universität Konstanz, Germany
}

(Received 12 August 2004; revised manuscript received 22 December 2004; published 28 April 2005)

\begin{abstract}
Multilayers of superconducting/ferromagnetic ( $\mathrm{SC} / \mathrm{FM}$ ) $\mathrm{YBa}_{2} \mathrm{Cu}_{3} \mathrm{O}_{7} / \mathrm{La}_{2 / 3} \mathrm{Ca}_{1 / 3} \mathrm{MnO}_{3}$ have been studied by neutron reflectometry. The occurrence of a structurally forbidden Bragg peak in the FM state highlights a significant difference between the nuclear and magnetic depth profiles. From comparison with simulated reflectivity curves we identify two possible magnetization profiles: (i) a magnetic moment within the SC layer antiparallel to the one in the FM layer (antiphase magnetic proximity effect) or (ii) a "dead" region in the FM layer with zero net magnetic moment. In addition, we observe an anomalous enhancement of the off-specular reflection in the SC state which signals a strong mutual interaction between SC and FM order parameters.
\end{abstract}

\section{DOI: 10.1103/PhysRevB.71.140509}

Recent advances in fabrication and characterization of multilayers with nanoscale periodicity based on perovskite oxides have opened an avenue in the investigation of materials with strong electron correlations. ${ }^{1}$ Superlattices composed of ferromagnets (FM) and superconductors (SC) are of particular interest because their mutually exclusive groundstate properties can give rise to unique quantum phenomena. ${ }^{2}$ Prominent examples are the so-called $\pi$-junction effect, ${ }^{3}$ where the phase of the SC order parameter is modulated across the layers, or states with a spatial modulation of the amplitudes of the FM and SC order parameters, such as spontaneous vortex phases or the Larkin-Ovchinikov-FuldeFerrel (LOFF) state. Experimental signatures include a nontrivial dependence of $T_{c}$ on the FM layer thickness ${ }^{4,5}$ and a complex magnetic phase diagram with reentrant SC states.

The work on the perovskite oxide FM/SC superlattices is motivated by the appealing properties of the cuprate high- $T_{c}$ superconductors (HTSC) whose high SC critical temperatures make them potentially useful for technological applications. Further, since HTSC are believed to be susceptible to a variety of competing instabilities, there is a high potential for $\mathrm{SC} / \mathrm{FM}$ quantum states in multilayer structures. This research is in its early stage, and relatively little is known about the nature of magnetism at the interface, the spatial distribution of the magnetization throughout the layers, and the interplay of FM and SC order parameters in general. Neutron reflectivity has been a tool of choice in investigating interfaces in thin films and multilayers. ${ }^{6,7}$ In general, it allows one to probe a potential normal to the surface which consists of the contributions from the atomic nuclei $V_{\text {nuc }}(z)$ and the magnetic potential $V_{\mathrm{mag}}(z)$. Since reflection takes place only at the interfaces of a multilayer, one can apply Bragg's law for a one-dimensional crystal to obtain the condition for constructive and destructive interference. In addition, one needs to consider that the response of subsequent interfaces contains a phase shift of $\pi$. From the Bragg peaks one obtains information on the number of layers from the peak width, the period length from the distance between adjacent peaks, and the ratio of the individual layer thicknesses from the relative peak intensities. In addition, the in-plane off-specular width of the peaks yields information about the lateral correlation length of the multilayers. This technique has provided valu-
PACS number(s): 74.78.Fk, 73.21.Ac, 74.45.+c, 74.81.-g

able information on the microscopic magnetic properties of classical FM/SC multilayers, but has so far not been successfully applied to perovskite oxide FM/SC multilayers.

In this Communication we report polarized and unpolarized neutron reflectivity measurements on symmetric superlattices [with identical $\mathrm{La}_{2 / 3} \mathrm{Ca}_{1 / 3} \mathrm{MnO}_{3}$ (LCMO) and $\mathrm{YBa}_{2} \mathrm{Cu}_{3} \mathrm{O}_{7}$ (YBCO) layer thicknesses] that consist of alternating layers of the FM colossal magnetoresistance material LCMO and the HTSC compound YBCO. Symmetric superlattices are well suited to explore a possible interference between SC and FM order parameters, because an extinction rule disallows all even-order Bragg reflections if $V_{\text {mag }}(z)$ is spatially uniform and confined to the LCMO layer. The reflectivity curves above the FM and $\mathrm{SC}$ transitions $\left(T>T_{\text {mag }}\right.$ and $T_{\mathrm{sc}}$ ) indeed exhibit only odd-numbered Bragg peaks and testify to the high structural quality of our superlattices (with an rms interface roughness ${ }^{7}$ of $\sigma \approx 5 \AA$ ). The reflectivity curves exhibit marked changes in the FM state as well as in the SC state. In particular, the appearance of a second-order magnetic Bragg peak below $T_{\text {mag }}$ indicates that $V_{\text {mag }}(z)$ either reaches into the YBCO layer with antiferromagnetic coupling across the interface (antiphase magnetic proximity scenario) or is confined to a spatial range significantly less than the thickness of the LCMO layer (dead layer scenario). Both scenarios are incompatible with a conventional magnetic proximity effect as proposed in Refs. 8,9. Furthermore, an anomalous enhancement of the off-specular reflection in the SC state indicates a strong mutual interaction of SC and FM order parameters.

Superlattices of $[\mathrm{LCMO}(98 \AA) / \mathrm{YBCO}(98 \AA)]_{7}$ (sample 1) and $[\operatorname{LCMO}(160 \AA) / \operatorname{YBCO}(160 \AA)]_{6}$ (sample 2) were grown by pulsed laser deposition (PLD) on $10 \times 10$ $\times 0.5 \mathrm{~mm}^{3} \mathrm{SrTiO}_{3}(001)$ substrates. ${ }^{5}$ Their high quality was confirmed by $\mathrm{x}$-ray diffraction, which showed epitaxial growth with the $c$ axis along (001). Resistivity and superconducting quantum interference device (SQUID) magnetization measurements revealed a FM transition at $T_{\text {mag }} \approx 165 \mathrm{~K}$ and a SC one at $T_{\mathrm{sc}} \approx 75 \mathrm{~K}$. Both values are substantially reduced from the typical bulk ones of $T_{\mathrm{mag}}^{\mathrm{LCMO}}=270 \mathrm{~K}$ and $T_{\mathrm{sc}}^{\mathrm{YBCO}}$ $=93 \mathrm{~K}$, likely due to the proximity effect. ${ }^{4,5}$

Unpolarized and polarized angle-dispersive neutron reflectivity measurements have been performed on the 2-axes 

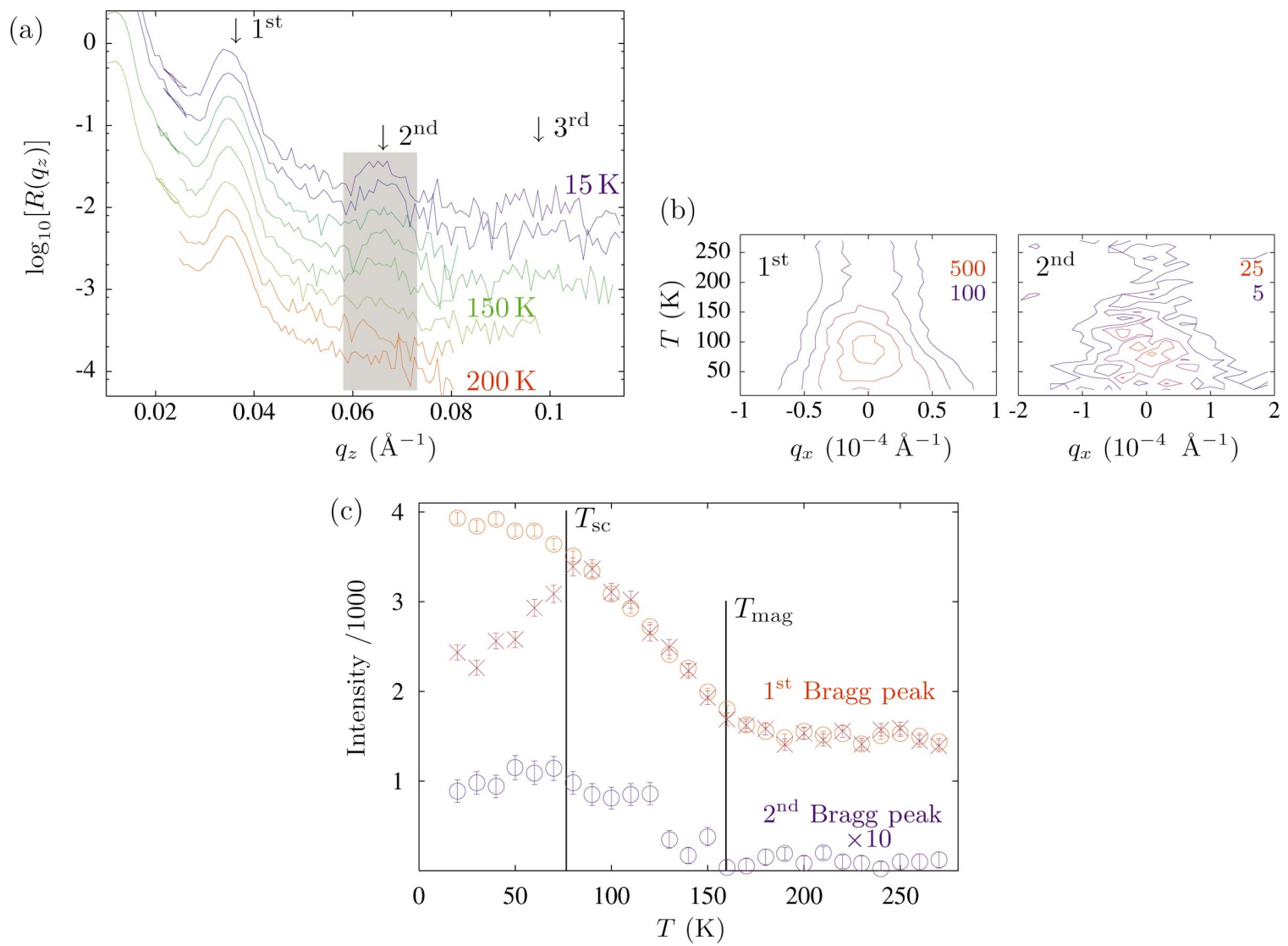

FIG. 1. (Color) (a) Specular reflectivity of sample 1 at 200, 170, 150, 120, 100, 70, 50, and $15 \mathrm{~K}$, for $H_{\text {ext }}=100$ Oe (field cooled). Curves are offset for clarity. Bragg peaks are marked by arrows. (b) $I\left(q_{x}, T\right)$ map for the first (left) and second (right) Bragg peaks. (c) $T$ dependence of the first (red) and second (blue) Bragg-peak intensities, integrated over $q_{x}(\bigcirc)$ and at $q_{x}=0(\times$, scaled by 6$)$.

diffractometers Morpheus at SINQ and ADAM at ILL. In both cases a ${ }^{3} \mathrm{He}$ detector was used. The samples were mounted in a closed-cycle refrigerator with a temperature range from 12 to $300 \mathrm{~K}$. The external magnetic field $H_{\text {ext }}$ produced by Helmholtz coils was oriented perpendicular to the scattering plane and parallel to the film surface.

Figure 1(a) displays unpolarized neutron reflectivity curves taken under specular condition. For the curves at $T$ $>T_{\text {mag }}$ (where only nuclear scattering contributes) the first Bragg peak is clearly visible at $q_{z} \approx 0.035 \AA^{-1}$, whereas the second Bragg peak at $q_{z} \approx 0.07 \AA^{-1}$ (as marked by the shaded area) is absent to within the noise level. This extinction of even-order peaks is expected due to the 1:1 layer thickness of the present SL where each period (unit cell) contains two interfaces whose response is phase shifted by a factor of $\pi$. Remarkable changes occur in the ferromagnetic state for $T<T_{\mathrm{mag}}$. Firstly, the intensity of the first Bragg peak (averaged over the neutron spin states) exhibits a sizable increase. This shows that the magnetic potential $V_{\text {mag }}(z)$ enhances the contrast between the YBCO and LCMO layers, as expected due to the onset of magnetic order in LCMO. Secondly, the signature of the structurally forbidden second Bragg peak suddenly appears in the spectra below $T_{\text {mag }}$. The magnetic origin of the second Bragg peak (confirmed by the polarized-beam data below) is indicative of a substantial difference between the spatial profiles of the nuclear and magnetic potentials. Another remarkable anomaly occurs in the vicinity of the SC transition. It is best seen in the off-specular rocking scans at the Bragg positions, as shown in Figs. 1(b) and 1(c). The off-specular reflectivity is sensitive to a momentum transfer parallel to the plane of the multilayer $\left(q_{x}\right)$ and thus provides information on in-plane correlation of the nuclear and magnetic profiles. The left panel of Fig. 1(b) shows that the off-specular scattering is weak and nearly temperature independent for $T>T_{\mathrm{mag}}$. Such diffuse scattering is characteristic of uncorrelated in-plane roughness of the nuclear potential. Below $T_{\text {mag }}$ the intensity of the specular and off-specular components increases continuously with no indication for a substantial change in the peak width. Below $T_{\mathrm{sc}}$, however, a pronounced broadening occurs, and weight is transferred from the specular to the off-specular part. This is readily visible in Fig. 1(c) where the specular intensity (crosses) of the first Bragg peak exhibits a strong decrease, whereas the intensity integrated along $q_{x}$ (circles) increases. The observed trend is indicative of a profound SC-induced increase in the magnetic roughness. A corresponding trend is observed for the second Bragg peak as shown in the right panel of Fig. 1(b). For the first Bragg peak we estimate that the full width at half maximum $\Delta q_{x}$ changes from 0.6 $\times 10^{-4} \AA^{-1}$ at $75 \mathrm{~K}$ to about $1.2 \times 10^{-4} \AA^{-1}$ at $15 \mathrm{~K}$. This translates into a change of the lateral correlation length from more than $15 \mu \mathrm{m}$ at $T \simeq T_{\text {sc }}$ to about $7 \mu \mathrm{m}$ at $T \ll T_{\text {sc }}$. These pronounced SC-induced changes of the in-plane component of the magnetic profile are suggestive of a sizable proximity 

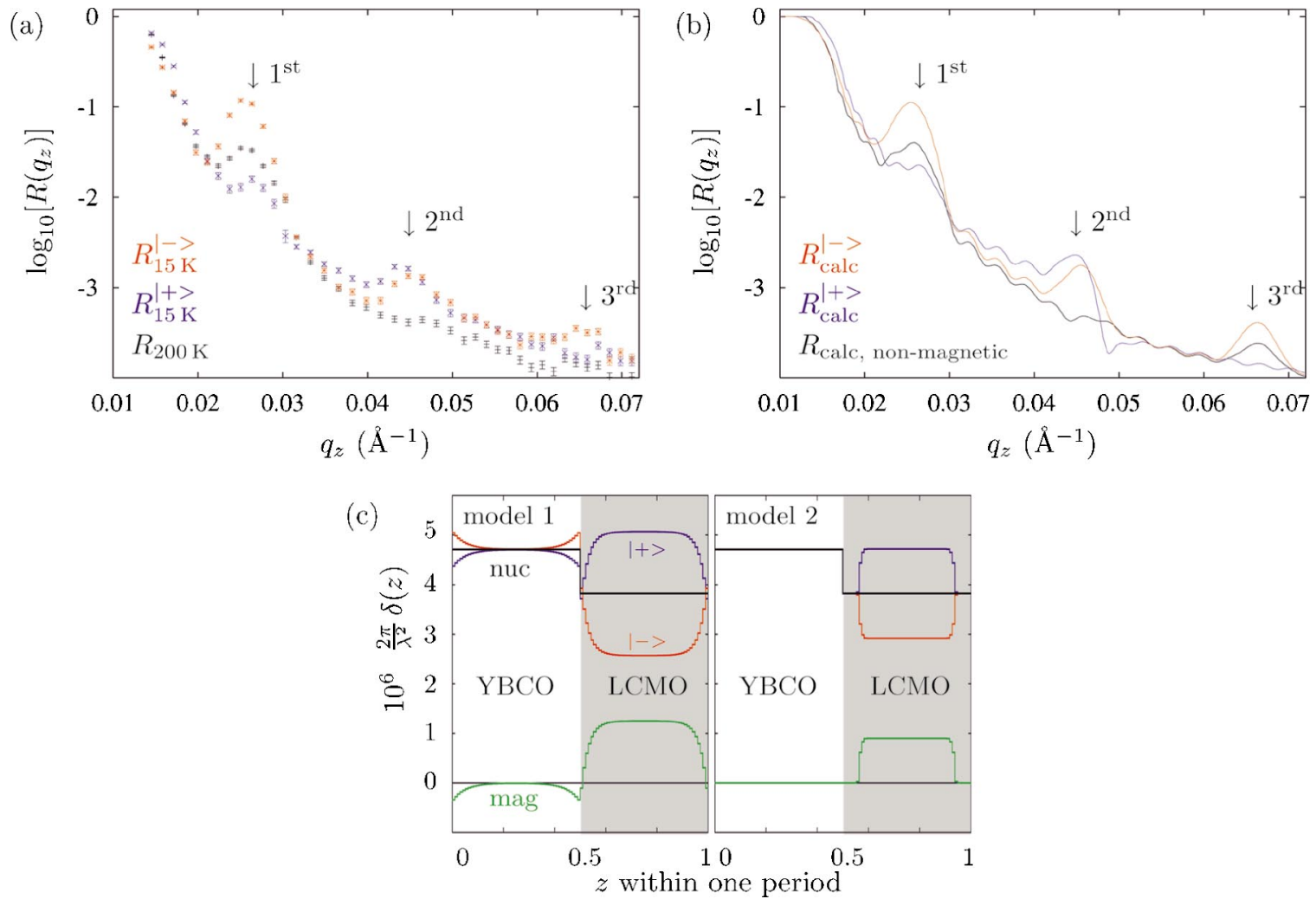

FIG. 2. (Color) (a) Polarized specular reflectivity of sample 2 at 200 and $15 \mathrm{~K}$, for $H_{\text {ext }}=100$ Oe (field cooled). (b) Simulated reflectivity curves (model 1) and (c) model potentials that reproduce the experimental data. Left: antiphase magnetic proximity effect (model 1); Right: "dead layer" (model 2). $\delta(z) \propto V(z)$ is the deviation of the refractive index from $1, \lambda$ is the neutron wavelength.

coupling of the SC and FM order parameters. SQUID magnetization data (not shown) indicate that the FM magnetic moments are oriented parallel to the layers of our superlattices. Orbital effects of the magnetic field in the SC layers are therefore expected to be weak, and the dominant interaction is the magnetic exchange coupling. This introduces a spin splitting of the electronic states and reduces the SC condensation energy. In return, the development of the SC order parameter favors the formation of FM domain boundaries where the pair breaking is substantially reduced. ${ }^{10}$ The anomalous decrease in the size of the FM domains therefore is indicative of a strong proximity coupling between the SC and FM order parameters. A spontaneous vortex phase (due to a minor perpendicular component of the FM moments) in the SC layers or the presence of an unconventional SC order parameter with a spin-triplet component could also contribute to the off-specular signal. ${ }^{11}$ These scenarios could be tested by mapping out the off-specular signal in further experiments with an improved signal-to-noise ratio.

We now describe a quantitative analysis of the magnetization profile perpendicular to the layers. We tested numerous models with the $\mathrm{x}$-ray and neutron reflectivity simulation $\operatorname{code}^{7}$ (EDXR) to compare the calculated reflectivity curves with the experimental ones. In order to separate structural and magnetic contributions, and to determine the quality of the interface, the nuclear contribution $V_{\text {nuc }}(z)$ was determined from the curves at $T>T_{\text {mag }}$. The obtained individual layer thicknesses are 98 and $160 \AA$, respectively, for samples 1 and 2 . The density for LCMO was reduced by $2 \%$ with respect to the bulk value. The interface was described by a roughness of $\sigma \approx 5 \AA$, which testifies to the high quality of our superlattices. It is well known that neutron reflectivity curves lack phase information and thus cannot be uniquely assigned to a particular density or magnetization profile. Nevertheless, we are able to identify only two possible solutions. The main challenge in selecting an appropriate magnetization profile is to reproduce the well-defined first structural Bragg peak, the magnetically induced second Bragg peak, and the low intensity of the third structural Bragg peak. An extra constraint is imposed by the marked differences in polarized up-spin and down-spin reflectivities as shown in Fig. 2(a). The presence of the second peak demonstrates that the magnetic potential $V_{\text {mag }}(z)$ cannot simply follow the blocklike nuclear profile. Accordingly, in the calculations we allowed for a modification of the magnetic potential by breaking down the unit cell into 96 sublayers. Based on extensive computer simulations of the specular reflectivity, we were able to exclude several physically meaningful models: (i) An antiferromagnetic coupling between the ferromagnetic layers would lead to a doubled period and hence to additional Bragg peaks, at $q_{z}=0.022 \AA$ and at $q_{z}=0.053 \AA$, which are not observed. (ii) A magnetic roughness of any length scale only leads to a faster decay of the reflectivity but not to a second Bragg peak. (iii) A conventional magnetic proximity effect where the magnetization exhibits an exponential decay into the SC layer also fails to describe our spectra.

In the following we discuss the only two successful models whose magnetization profiles are illustrated in Fig. 2(c). Model 1 (left panel) assumes a sizable magnetic moment 
within the YBCO layer that couples antiferromagnetically to the one in LCMO (antiphase magnetic proximity effect). Notably, the antiparallel alignment is essential to reproduce the observed second Bragg-peak positions and intensities. Model 2 (right panel) contains a "dead" region with no net magnetic moment (either paramagnetic or antiferromagnetic) within the LCMO layer. The resulting fits of the polarized reflectivities using model 1 are shown in Fig. 2(b). The values of the magnetic induction thus obtained are $B_{\mathrm{YBCO}}=0.6 \mathrm{~T}$ and $B_{\mathrm{LCMO}}=0.9 \mathrm{~T}$ (sample 1 ), and $B_{\mathrm{YBCO}}=0.6 \mathrm{~T}$ and $B_{\mathrm{LCMO}}$ $=1.4 \mathrm{~T}$ (sample 2). The penetration depth of the interfacial magnetization profile is of the order of $20 \AA$ in the YBCO layer, and $10 \AA$ in the LCMO layer. Similar fits were obtained with model 2 (not shown) assuming a thickness of the "dead layer" of about $20 \AA$ and magnetic induction $B$ of 1.1 $\mathrm{T}$ (sample 1) and 1.5 T (sample 2).

The calculations do not allow one to differentiate between these two models. Furthermore, both models can be justified on physical grounds. The magnetically "dead layer" in LCMO, the centerpiece of model 2, could arise from interfacial strain or charge transfer across the interface. According to the phase diagram of LCMO, this could introduce an insulating layer with antiferromagnetic order. Alternatively, it could arise from interfacial disorder such as intergrowth or interdiffusion of cations. However, the latter explanation seems unlikely since the combined results of x-ray diffraction, electron microscopy, ${ }^{5}$ and in particular, neutron reflectivity curves for $T>T_{\text {mag }}$, testify to the high quality of the interfaces with practically absent intergrowth and small overall structural roughness of the order of $5 \AA$.

In terms of interesting physical phenomena, the scenario of model 1 is far more appealing. Its essential feature, that is, a thin layer on the SC side which has a net magnetic moment oriented antiparallel to the one in the FM layer, has recently been proposed theoretically. ${ }^{12}$ The unusual magnetization profile near the interface originates here from Cooper pairs that have a finite overlap with both the FM and the SC layers. Heuristically, the preferential spin alignment of one electron in the FM layer leads to an antiparallel spin orientation of the second electron of the spin-singlet pair that resides in the SC layer. One may argue that this model cannot be relevant since the second Bragg peak appears already well above $T_{\text {sc }}$ while Cooper pairs exist only for $T<T_{c}$. Nevertheless, a possible explanation may be found in terms of strong SC fluctuations above the macroscopic transition temperature which are well known to be prominent in underdoped HTSC. Alternatively, one may speculate that corresponding arguments, as outlined in Ref 12 for the case of Cooper pairs, may be applicable for other kinds of itinerant spin-singlet pairs. The possibility of spin-singlet pairs at elevated temperatures $T>T_{\text {sc }}$ has been indeed proposed in the context of the unusual normal-state electronic properties of the cuprate HTSC (so-called pseudogap phenomenon). ${ }^{13} \mathrm{Fi}$ nally, we note that our data are consistent with recent macroscopic magnetization measurements suggesting an antiferromagnetic component of the magnetization profile at the YBCO/LCMO interface. ${ }^{14}$ Underdoped cuprates are known to be susceptible to antiferromagnetic order, and a staggered magnetization profile whose amplitude decreases as a function of distance from the interface would generate a net magnetization in YBCO, as observed.

In summary, our neutron reflectometry measurements on high-quality $\mathrm{YBa}_{2} \mathrm{Cu}_{3} \mathrm{O}_{7} / \mathrm{La}_{2 / 3} \mathrm{Ca}_{1 / 3} \mathrm{MnO}_{3}$ multilayers have revealed detailed, microscopic information about the magnetization profile as a function of in-plane and out-of-plane wave vectors. First of all, our data testify for the high structural quality of our multilayers with an rms interface roughness $^{7}$ of $\sigma \approx 5 \AA$. Furthermore, we observed characteristic differences between the nuclear and the magnetic scattering profiles which allowed us to identify two possible scenarios of (i) an antiphase magnetic proximity coupling where a FM moment is induced in YBCO that is oriented antiparallel to the one in LCMO, and (ii) a ferromagnetically "dead layer" (either paramagnetic or antiferromagnetic) within the LCMO region. Finally, we observed an anomalous enhancement of the off-specular reflection in the SC state which suggests a strong mutual interaction between the $\mathrm{SC}$ and the FM order parameters.

We acknowledge M. Wolff for support on ADAM at ILL, France. This work was partly performed on Morpheus at SINQ, Paul Scherrer Institute, Switzerland.

${ }^{8}$ C. A. R. Sá de Melo, Phys. Rev. Lett. 79, 1933 (1997).

${ }^{9}$ Z. Radovic, L. Dobrosavljevic-Grujic, A. I. Buzdin, and J. R. Clem, Phys. Rev. B 38, 2388 (1988); Z. Radovic, M. Ledvij, L. Dobrosavljevic-Grujic, A. I. Buzdin, and J. R. Clem ibid. 44, 759 (1991).

${ }^{10}$ A. I. Buzdin and L. N. Bulaevskii, Sov. Phys. JETP 67, 576 (1988); F. S. Bergeret, K. B. Efetov, and A. I. Larkin, Phys. Rev. B 62, 11872 (2000).

${ }^{11}$ A. I. Buzdin and A. S. Melnikov, Phys. Rev. B 67, 020503(R) (2003); A. F. Volkov, F. S. Bergeret, and K. B. Efetov, Phys. Rev. Lett. 90, 117006 (2003).

${ }^{12}$ F. S. Bergeret, A. F. Volkov, and K. B. Efetov, Phys. Rev. B 69, 174504 (2004).

${ }^{13}$ P. W. Anderson, Science 235, 1196 (1987); P. W. Anderson, The Theory of Superconductivity in the High- $T_{c}$ Cuprates, (Princeton University Press, Princeton, NJ, 1997).

${ }^{14}$ N. Haberkorn et al., Appl. Phys. Lett. 84, 3927 (2004); P. Przyslupski et al., Phys. Rev. B 69, 134428 (2004). 